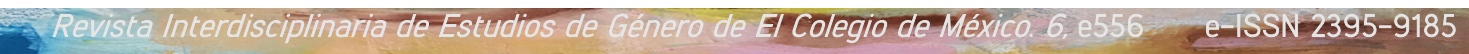

http://dx.doi.org/10.24201/reg.v6i0.556

Artículo

\title{
Las universidades frente a la violencia de género. \\ El caso de la Universidad Autónoma de Guanajuato
}

\section{Universities Against Gender Violence. \\ The Case of University of Guanajuato}

Recibido: 26 de noviembre de 2019

Aceptado: 5 de junio de 2020

Publicado: 20 de julio de 2020

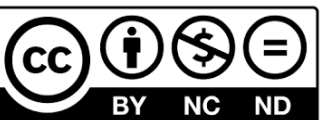

Esta obra está protegida bajo una Licencia Creative Commons AtribuciónNoComercial-SinDerivadas 4.0

Internacional (CC BY-NC-ND 4.0)
Helena Varela Guinot

Universidad Iberoamericana, \#Red Politólogas México, Ciudad de México, México. email: helena.varela@ibero.mx, (0) https://orcid.org/0000-0002-2166-2316

\section{Resumen}

Se analiza cómo las universidades están actuando frente a la violencia de género. A partir de la propuesta de la teoría fundamentada, se tomó el caso de la denuncia que realizó en 2018 un grupo de estudiantes de la Universidad Autónoma de Guanajuato. A través de entrevistas en profundidad semiestructuradas a autoridades, estudiantes y docentes, se presenta el proceso institucional, con el fin de identificar sus alcances y limitaciones. Se muestra que las autoridades actúan de manera reactiva, con escasas medidas de prevención de la violencia. La falta de una mirada integral y sistemática da como resultado respuestas erráticas y apresuradas, lo cual provoca un malestar generalizado en la comunidad universitaria, sin que haya una atención de fondo a la problemática.

CÓMO CITAR: Varela, Helena. (2020). Las universidades frente a la violencia de género. El caso de la Universidad Autónoma de Guanajuato. Revista Interdisciplinaria de Estudios de Género de El Colegio de México, 6, e556. doi: http://dx.doi.org/10.24201/reg.v6i0.556

http://estudiosdegenero.colmex.mx 
Palabras clave: violencia de género; universidades; perspectiva de género; teoría fundamentada.

\begin{abstract}
This article analyzes how universities are addressing gender violence. Using the grounded theory, the author studied the case of the complaint made in 2018 by a group of students from the University of Guanajuato. A series in-depth, semi-structured interviews with representatives from the academic administration, students and teachers, recorded the institutional process in order to identify its scope and limitations. The findings show that the administration acts reactively, with few measures to prevent violence. A piecemeal approach leads to erratic and hasty responses, that cause widespread concern whith in the university community, and the problema is never tackled properly.
\end{abstract}

Key words: gender violence; universities; gender perspective; grounded theory.

\title{
Introducción
}

En marzo de 2019, a través del hashtag \#MeToo, inició un movimiento donde se denunciaban, anónimamente, casos de acoso y violencia contra las mujeres ${ }^{1}$; en México se vivió una etapa de convulsión, al extenderse a ámbitos específicos como el del cine, la música, la literatura, el periodismo o la academia. En el caso de \#MeTooAcadémicos, en

\footnotetext{
${ }^{1}$ Para efectos de esta investigación, se toma la definición de violencia contra la mujer que se establece en la Ley General de Acceso de las Mujeres a una Vida Libre de Violencia, en su artículo 5, IV, que la define como "Cualquier acción u omisión, basada en su género, que les cause daño o sufrimiento psicológico, físico, patrimonial, económico, sexual o la muerte tanto en el ámbito privado como en el público”. Asimismo, en el artículo 13 se establece la diferencia entre hostigamiento sexual ("es el ejercicio del poder, en una relación de subordinación real de la víctima frente al agresor en los ámbitos laboral y/o escolar. Se expresa en conductas verbales, físicas o ambas, relacionadas con la sexualidad de connotación lasciva") y el acoso sexual ("es una forma de violencia en la que, si bien no existe la subordinación, hay un ejercicio abusivo de poder que conlleva a un estado de indefensión y de riesgo para la víctima, independientemente de que se realice en uno o varios eventos"). Recuperado de http://www.diputados.gob.mx/LeyesBiblio/pdf/LGAMVLV_130418.pdf
} 
menos de un mes se habían recibido denuncias contra 349 personas (36 pertenecientes al nivel medio superior y 313 a universidades) ${ }^{2}$. Este tipo de expresiones puso de manifiesto tres cuestiones fundamentales: en primer lugar, que el fenómeno de la violencia de género está ampliamente extendido en nuestra sociedad y, por lo tanto, se hace presente en los diferentes espacios, incluyendo el educativo. En segundo lugar, la violencia contra las mujeres tiene múltiples maneras de manifestarse, aunque el nivel de impacto no es el mismo: un chiste sexista, una mirada lasciva, una amenaza, un golpe, una violación, pueden dejar huellas distintas, pero responden a un patrón común de relaciones de dominación y dependencia entre dos grupos - los victimarios y las víctimas_-, "cuando uno se apropia de los derechos y privilegios del otro y fija sus límites" (Apfelbaum, 1989, p.5). En el caso de las universidades, estas formas de dominación adquieren especial relevancia en las interacciones estudiantado-profesorado porque la asimetría se profundiza por la relación jerárquica existente. En tercer lugar, el movimiento \#MeTooAcadémicos evidenció que actualmente los canales formales e institucionales de denuncia no están sirviendo; la percepción de que no hay garantías de certidumbre y seguridad hace que las denuncias presentadas formalmente sean pocas en comparación con las que se presentan a través de mecanismos informales ${ }^{3}$.

Sin embargo, como ha señalado Daniela Cerva (2019) en su investigación sobre el activismo feminista en las universidades, a pesar de que no se confía en las autoridades ni en la vía institucional, ésta sigue siendo una opción utilizada para canalizar sus demandas:

Ellas [las estudiantes activistas] no están posicionándose fuera del sistema, todo lo contrario, creen que la principal vía de atención a sus demandas es dar certeza normativa, tanto en los procesos de denuncia como en la sanción y alejamiento del victimario de los centros de estudio (Cerva, 2019, p.20).

\footnotetext{
${ }^{2}$ Información obtenida del Twitter \#MeTooAcadémicos, recuperado de https://twitter.com/metooacademicos

${ }^{3}$ En 2011, Laura Hensley Choate daba cuenta de diferentes estudios que se habían realizado en Estados Unidos, en donde se señalaba la poca propensión a denunciar la violencia sexual en las universidades, debido a que se consideraba que la institución no apoyaría a las víctimas ni las tomaría realmente en serio (Hensley, 2011, p.166). Para el caso mexicano, Barreto da cuenta de estudios que muestran "la poca frecuencia de denuncias en proporción al número de universitarias afectadas" (Barreto, 2017, 263).
} 
La presente investigación tiene por objetivo analizar por qué las universidades no están siendo capaces de atender adecuadamente el fenómeno de la violencia de género. Para ello, se utilizó una propuesta de metodología inductiva, basada en la teoría fundamentada de Glaser y Strauss. El abordaje es mediante un estudio de caso que se presentó en el Campus León de la Universidad Autónoma de Guanajuato (UG), en agosto de 2018, cuando un grupo de estudiantes (la Colectiva de Alumnas UG en Sororidad), con el apoyo de la Asociación Civil Las Libres, decidió exponer públicamente las situaciones de acoso que se viven en la universidad para, posteriormente, levantar una queja formal en la institución. Se examinará la manera en que fue atendido el caso, utilizando las herramientas del análisis de coyuntura: caracterización del contexto; identificación de los actores más relevantes según su posicionamiento frente al acontecimiento principal - la denuncia de las estudiantes - de acuerdo a las categorías establecidas en el Centro de Documentación e Información Bolivia (Cedib,2006) — protagonistas, ayudantes, opositores y destinatarios—; y análisis de la toma de decisiones en función de la correlación de fuerzas y del momento en que se fueron produciendo los hechos. Se prestará especial atención al papel desempeñado por las autoridades universitarias que, como se verá, oscilaron entre coadyuvar a la causa planteada por las estudiantes (tal y como lo establece la normatividad institucional) y frenar sus logros (por las decisiones tomadas en determinados momentos).

\section{Propuesta metodológica: la teoría fundamentada}

Desde que Fisher, Cullen y Turner (2000) dieron a conocer los resultados de su investigación sobre la violencia sexual en las universidades de Estados Unidos que advertían de las formas y el grado en que se presentaba este fenómeno, han ido surgiendo una serie de estudios en diferentes países sobre la temática. Valls, Oliver, Sánchez-Aroca, Ruiz, Melgar (2007), identifican distintos temas en los que se han centrado las investigaciones sobre violencia de género en universidades: los tipos de violencia que se presentan, el nivel de recurrencia, el grado en que la problemática es aceptada y reconocida por la misma población, los estudios que se centran en el papel de la universidad como generadora de violencia, los efectos o consecuencias sobre las personas afectadas, o el tema específico de las relaciones profesorado-estudiantado, mediadas por las relaciones de poder. Algunos ejemplos sobre 
trabajos realizados en diferentes países de América Latina son Nash (2015) para el caso de la Universidad de Chile; Castaño-Castrillón, González, Guzmán, Montoya, Murillo, PáezCala, Parra, Salazar y Velásquez (2010) que analiza el acoso sexual en la Universidad de Manizales (Colombia); Carvajal Orlich y Delvó Gutiérrez (2009) que se centran en el hostigamiento sexual en la universidad pública de Costa Rica; y Bardales Mendoza y Ortiz Miguel (2012) quienes realizan un estudio exploratorio sobre hostigamiento sexual en población universitaria del Perú.

En México, junto a la información de corte periodístico, también se ha ido generando conocimiento en torno a esta problemática, con diferentes perspectivas. Autoras como Buquet (2016) o Cerva (2017) se han centrado más en la perspectiva de género, en las condiciones de desigualdad, y en cómo el género influye en los diferentes procesos institucionales dentro de la universidad. La investigación de Carrillo Meraz (2015) analiza el caso de la Universidad Autónoma Metropolitana, pero aborda el tema de la violencia en términos generales, no en la cuestión específica de la violencia de género. Otros estudios de casos específicos de universidades son el de Castro y Vázquez (2008) que explican la violencia que sufren las estudiantes de la Universidad Autónoma de Chapingo, como reflejo de un proceso de "domesticación" que se inicia desde etapas tempranas de sus vidas; Gamboa Solís (2019) se enfoca en la eficacia del protocolo en la Universidad Michoacana de San Nicolás de Hidalgo; y Evangelista (2017) se centra en el sureste mexicano. Asimismo, destacan los trabajos compilados en Casillas, Dorantes y Ortiz (2017); así como las investigaciones sobre las movilizaciones de estudiantes y el impacto que han tenido en los procesos de visibilización de la problemática de Barreto y Flores (2016) y Barreto (2018).

Sin embargo, son relativamente escasas las investigaciones sobre la manera en que están actuando las universidades y los retos que están atravesando para hacer frente al fenómeno de la violencia de género. Hay que destacar el estudio de Barreto (2017) que explica el silencio institucional frente a la problemática, a partir de la sociología de la negación, tanto a nivel personal como colectivo; y el de Mingo y Moreno (2015) que entienden los conceptos del "derecho a no saber" y de "ignorancia cultivada" como medios para "eludir el reconocimiento de las distintas formas de violencia contra las mujeres que forman parte de 
la comunidad universitaria" (Mingo y Moreno, 2015, p.139). En ambos casos las autoras muestran la pasividad de las instituciones de educación superior frente a la violencia de género, que es "tolerada silenciosamente" (Barreto, 2017, p. 262). Sin embargo, es necesario ahondar en cómo operan estos mecanismos de negación y silencio, impidiendo una atención adecuada a la problemática (protección de las víctimas, sanción, reparación y garantía de no repetición). Por lo anterior, se optó por hacer un estudio de caso que permitiera identificar rasgos característicos de los procesos institucionales y la reacción de las autoridades frente a la violencia de género.

Una investigación de corte cualitativo permite percibir la gran variedad de matices y la complejidad del fenómeno que se estudia. Ante un suceso de violencia de género, en donde al menos dos grupos quedan explícitamente confrontados (los agresores frente a las agredidas), era de esperar que los puntos de vista fueran radicalmente distintos, incluso contradictorios entre sí. Para una buena comprensión, era importante considerar las múltiples miradas, experiencias y percepciones que conforman las distintas narrativas para identificar las categorías de análisis a partir de las cuales formular una serie de hipótesis.

La investigación se planteó desde la perspectiva de la teoría fundamentada, a través de entrevistas en profundidad semiestructuradas a informantes clave que intervinieron, directa o indirectamente, en los sucesos que se analizan, y que pertenecen a las distintas categorías de actores: estudiantes de la Colectiva (protagonistas), miembros del Centro Las Libres (ayudantes), profesores involucrados (opositores), otros profesores y profesoras de la Universidad (destinatarios) y autoridades universitarias (papel que se analizará en la investigación $)^{4}$. La idea que subyace es que "la mejor forma de representar la realidad social es mediante teorías obtenidas de los datos, y no con teorías elaboradas mediante la relación

\footnotetext{
${ }^{4}$ En total, se realizaron 16 entrevistas, 15 de las cuales fueron grabadas. Aun cuando algunas de las personas entrevistadas me dieron autorización para poner sus nombres, salvo en los casos en que se considera estrictamente necesario, se ha optado por mantener el anonimato, con el fin de centrar la atención en el proceso y en los aspectos institucionales, más que en las personas concretas. Para la codificación, se consideraron cuatro tipos de actores (autoridades, docentes, colectiva -que incluye a las integrantes de La Colectiva Las Libres-y denunciados). A cada persona entrevistada se la identificó por el nombre del grupo al que pertenece y un número o código asignado. Asimismo, al hacer referencia a la información de las entrevistas se especifica la fecha en que fueron realizadas.
} 
de una serie de conceptos basados en la especulación" (Andréu Abela, García-Nieto y Pérez Corbacho, 2007, p.55).

Conforme se fue codificando la información obtenida en las entrevistas, comparando y contrastando los puntos de vista, se pudieron formular las primeras hipótesis de la teoría sustantiva, que aparecen planteadas en el apartado de reflexiones finales ${ }^{5}$.

\section{El contexto regional y universitario}

Guanajuato es un estado del centro de México con una población total, de acuerdo con el censo de 2015, de 5853677 habitantes, la ciudad de León es, con algo más de millón y medio de habitantes, la más poblada del estado (INEGI, 2017). En comparación con otras entidades federativas, se encuentra en el tercio con el índice de desarrollo humano más bajo de la república (en el 2012 ocupaba el lugar número 26, con un índice de 0.720), pero la ciudad de León (con un 0.776) se encuentra por encima del promedio nacional (PNUD, 2019).

Más allá de los datos sociodemográficos, uno de los datos más característicos de la sociedad guanajuatense es su carácter altamente conservador, que no sólo se ha visto reflejado en las ideas políticas, sino también en aspectos morales o religiosos. Desde el punto de vista político, llama la atención que desde 1991, año en que llega a la gubernatura Carlos Medina Plascencia, todos los gobernadores han sido del Partido Acción Nacional (PAN), es uno de los estados que menos alternancia ha sufrido en el período democrático. De la misma manera, la presidencia municipal de León también ha estado en manos del PAN desde los años noventa, excepto en el breve período del 2012 al 2015 en el que la encabezó el Partido de la Revolución Institucional (PRI).

Aun cuando el PAN no es un ente monolítico, ha sido consistente en su postura hacia ciertos temas, más todavía si se considera la fuerte presencia que tiene en la región la organización

\footnotetext{
${ }^{5}$ La aplicación de la metodología se ha realizado a partir de Andréu Abela, García-Nieto y Pérez Corbacho (2007) y Trinidad, Carrero y Soriano (2006). 
ultracatólica El Yunque, muy ligada a posiciones ultraconservadoras, como señala Arriola (1994) los temas en los que el partido recibe el calificativo de "conservador y reaccionario" son los que tienen que ver con la educación, la Iglesia y el estado. Esto hace que el partido “en las propuestas políticas actúa como partido moderno y en los temas relacionados con la moral personal, como uno doctrinario" (Aguilar, 6 de octubre de 2013). Ya en los años cincuenta, Carlos Fuentes daba cuenta del carácter específico del guanajuatense:

Así, el guanajuatense es un mocho calificado. Un mocho laico (como todos los eficaces) capaz de servir a la iglesia más oportuna y que, en su concepto, garantice la mejor administración práctica de la "voluntad general" teórica. Inteligentes, de propósitos internos claros y manera exterior velada, herederos de una tradición que el excesivo centralismo mexicano no ha alcanzado a destruir, los guanajuatenses representan la cima del espíritu del centro de la República (Fuentes, 1959, pp.24-25).

Esta visión conservadora, en donde "la base de la vida gira en torno a las directrices dadas por la Iglesia católica, la costumbre, la tradición y las convenciones sociales” (Rionda, 2013) va a ser un factor importante para entender el caso que se está analizando y la manera cómo los diferentes actores reaccionaron frente al mismo. Llama la atención que, sin ser un tema explícitamente abordado en las entrevistas, el conservadurismo, fue recurrentemente mencionado y tomado en cuenta para la formulación de las hipótesis. Para algunas personas, el conservadurismo del estado explica por qué a pesar de que el fenómeno del acoso era conocido en la universidad se había hecho tan poco, y por qué, incluso, cuando estalla el caso reciben pocas muestras de apoyo: “de Guanajuato se refleja mucho en los estudiantes de aquí, por ejemplo, la nula tradición de protesta, de lucha estudiantil, de organización. Antes de nosotras, nadie se organizaba para nada, ni rendición de cuentas, nada" (Colectiva 3, comunicación personal, 17 de mayo de 2019). En otros casos, se menciona cómo la forma de ser del guanajuatense tiende a evitar problemas, mirar para otro lado y hacer como si nada estuviera ocurriendo, que encaja perfectamente con la estrategia del "derecho a no saber" (ignorar lo que está ocurriendo, no darle la importancia debida, o considerarlo como un problema individual de una persona, y no como una cuestión social) y de la "ignorancia 
cultivada" (resistirse a entender lo que implica la violencia de género), que mencionan Mingo y Moreno (2015). Es más, hay quien va más allá, y señala que en la idiosincrasia está también el reaccionar ante la problemática con estrategias muy pragmáticas (en la línea de lo que ya señalaba Carlos Fuentes), poco confrontativas, buscando no alterar el statu quo.

Esto no es Guerrero, de alguna manera eso hace que haya una tendencia al gatopardismo, que todo cambie para que todo siga igual; que tenemos que hacer una cosa de género, pues la hacemos, pero con calma, con cuidado, no afecten demasiados intereses, no toquen demasiadas cosas, no quieras democratizar la Universidad, que es muy vertical (Docentes 1, comunicación personal, 15 de mayo de 2019).

Más adelante se explicará sobre el peso que pudo tener este factor, para explicar la manera en que se fueron dando los acontecimientos y cómo fueron actuando las personas involucradas.

Al contexto regional hay que añadir el propio contexto de la Universidad Autónoma de Guanajuato, una universidad que, como dijeron las personas entrevistadas, se caracteriza por un nivel de verticalidad muy fuerte en su forma de organización, en donde los procesos se dan de arriba para abajo, y siempre a través de intermediaciones de las autoridades. Esto propicia la conformación de grupos de poder, con una serie de prácticas que quedan en ámbitos opacos, propiciando la discrecionalidad e, incluso, según señalaron algunos de los testimonios, la corrupción. Algunas de las cuestiones que se señalaron pueden ser difíciles de probar, pero llama la atención que las propias autoridades reconocen que existe la percepción de que hay estos grupos de poder y amiguismo: "Estás en la universidad de Guanajuato. El ingrediente adicional es que se protegen entre ellos por una especie de afiliación política e ideológica” (Docentes 3, comunicación personal, 16 de mayo de 2019).

Incluso, se habla de un "sistema de amafiamiento de la organización del poder", conformado por "inatrapables" (Docentes 4, comunicación personal, 17 de mayo de 2019), en donde se 
percibía que "todo lo ganaban ellos, todo lo tienen ellos, ellos metían a su gente" (Docentes 5, comunicación personal, 15 de mayo de 2019).

Creo que hay muchas redes de complicidad y mucha corrupción, entonces me parece que, como dicen los dichos populares, que son muy sabios, les conocemos cosas, unos conocen cosas de otros y entonces, mejor así. Por eso funciona muy bien la complicidad (Colectiva 4, comunicación personal, 16 de mayo de 2019).

Cuando se preguntó a una de las personas entrevistadas, perteneciente al grupo de Autoridades, si existía este tipo de grupos de poder que buscaban protegerse entre ellos, contestó de una manera evasiva, pero al mismo tiempo, muy significativa: "Yo no puedo asegurar, no es una afirmación que yo puedo respaldar, pero no sería raro que ocurriera" (Autoridades 1, 19 de mayo de 2019).

Barreto (2017) menciona que las tradiciones de lealtad imperantes en las universidades pueden contribuir a fortalecer ese clima de silencio institucional. Son estas redes y grupos de poder los que permiten que muchas veces se solapen entre sí, e impidan que las denuncias prosperen. Esto a su vez, puede influir en el tipo de reacción que se suscita entre las denunciantes (desconfianza hacia los mecanismos formales, enojo, hartazgo) que puede propiciar otras formas de movilización (escraches, tendederos, denuncias públicas, etc.).

Finalmente, un último elemento de carácter coyuntural que hay que tener en cuenta para el análisis de los sucesos fue la posible ratificación del rector, proceso que estuvo gravitando en todo momento, durante los sucesos ${ }^{6}$. Para algunas personas entrevistadas, la reacción de la máxima autoridad para el caso que nos ocupa sólo puede ser explicada a partir de la coyuntura política específica de su próxima reelección: “Aquí en el caso de Guanajuato fue miedo, pues hay una coyuntura electoral; este año hay elecciones, estamos hablando que no

\footnotetext{
${ }^{6}$ A finales de agosto de 2019 se hizo pública la ratificación del rector Felipe Guerrero Agripino, para un segundo período de cuatro años.
} 
ha transcurrido un año, entonces, que al grupo en el poder le afectó..." (Denunciados 2, comunicación personal, 16 de mayo de 2019).

En resumen, si bien el conjunto de las instituciones de educación superior se enfrenta a problemas compartidos sobre las dificultades para erradicar la violencia de género ${ }^{7}$, en el caso de la denuncia de la Colectiva de la UG, la situación es más compleja por estar ubicada en una sociedad conservadora a la que le cuesta entender las demandas planteadas desde sectores más progresistas de la sociedad, como pueden ser los grupos feministas. Además, la opacidad y la existencia de grupos de poder en la universidad puede hacer más difícil atender la problemática, sobre todo si las personas acusadas son cercanas a las autoridades que tienen que tomar las decisiones. Todos estos elementos serán retomados más adelante.

\section{El caso Kala como antecedente del fenómeno de la violencia de género en la UG}

El caso de las acusaciones levantadas contra profesores de la Universidad de Guanajuato, en agosto de 2018, tiene un antecedente que ayuda a entender lo ocurrido: la denuncia en 2015 de una estudiante becaria en contra del entonces coordinador del Doctorado Interinstitucional en Derecho, Julio César Kala. La agresión había tenido lugar durante un viaje académico a Aguascalientes, y como resultado de ésta se interpusieron denuncias tanto en la Procuraduría de Justicia de Aguascalientes como en la de Guanajuato. En el primer caso, la Fiscalía General de Justicia de Aguascalientes archivó la causa por falta de pruebas; en el caso de Guanajuato, el ministerio público no vinculó a proceso debido a que los hechos habían ocurrido en otro estado. Poco tiempo después se supo que las autoridades de la universidad, dejándose llevar por el hecho de que en el sistema penal no se le había fincado ninguna responsabilidad al profesor, optaron por exonerarlo e incluso lo mantuvieron en su cargo y realizando sus actividades habituales.

El caso Kala puso en evidencia lo poco preparada que estaba la universidad para hacer frente a este tipo de escenarios relacionados con la violencia de género. En ese momento, no existía

\footnotetext{
${ }^{7}$ Muestra de ello es la proliferación de los "tendederos del acoso" en universidades públicas y privadas, como vía de expresión que visibiliza la violencia de género presente en las instituciones de educación superior, y la falta de respuesta por parte de las autoridades (ver, por ejemplo, Expansión Política, 20 de marzo de 2020).
} 
ningún protocolo que pudiera orientar a las personas responsables sobre cómo tenían que ser abordadas las denuncias, de qué manera debían ser atendidas las víctimas, y cuál era el proceso apropiado para intervenir sin caer en dinámicas de revictimización. Se mediaba de manera irreflexiva, porque no existía el conocimiento sobre la problemática ni la preparación para responder adecuadamente, de tal manera que las víctimas quedaban en una situación de desprotección y vulnerabilidad. Sin mecanismos pertinentes de atención y con personas que no han sido sensibilizadas en la perspectiva de género ni en atención a la violencia de género, era muy fácil actuar protegiendo al agresor y poniendo en entredicho la veracidad de la denuncia, aduciendo intereses ocultos (la estudiante se quiere vengar por alguna situación concreta y personal que tuvo con el profesor) o corresponsabilidad en la agresión (la estudiante lo provocó). Era el terreno ideal para la negación y el silencio institucional, con un juego de simulación que, en el mejor de los casos, aplicaba la táctica de "barrer bajo la alfombra" (Phips, 2018).

A raíz de la decisión de las autoridades de la universidad de no hacer algo al respecto, se desató una oleada de quejas contra la institución, que ponían en evidencia la impunidad y la tendencia a proteger al profesor frente a la denunciante. La propia víctima adujo que en varias ocasiones había recibido amenazas y chantajes desde distintas instancias, para que retirara su denuncia, y ello incluyó el hecho de que el acusado le ofreciera un cheque en blanco, a cambio de que cesaran las acusaciones. Como ella señala, este tipo de reacciones denotaba que "existe una estructura de poder y de pago de favores, sobre todo, en la Facultad de Derecho, donde los maestros son jueces y magistrados” (Álvarez, 2018).

El caso Kala es hoy todavía una herida abierta en la universidad, y se pudo constatar al realizar las entrevistas, pues fue un tema que todas las personas sacaron a colación de manera espontánea. Para las víctimas, es un claro ejemplo de cómo las autoridades se solapan entre sí, y prefieren protegerse, en lugar de hacer frente a la problemática. Esto propició la desconfianza hacia las instancias institucionales, que luego explicaría la reacción de las estudiantes de la Colectiva Alumnas UG en Sororidad, cuando decidieron hacer públicos los casos de acoso. Además, los sucesos de 2015 afianzaron la creencia de que, en realidad, los casos de acoso son conocidos por todo el mundo (no es algo que no se supiera), pero están 
aceptados y normalizados por una sociedad que opta por no afrontarlos abiertamente (mecanismo del “derecho a no saber”). Esa idea de que aquí todo el mundo sabía, pero nadie hacía nada, permeó en una parte de la comunidad universitaria. Así lo reconocen incluso las autoridades entrevistadas:

Nos falló interlocución, en ese sentido, o sea, generar los mecanismos de confianza y lo digo abiertamente, las alumnas no se acercaron porque me identificaron muy cerca de los profesores (Autoridades 2, comunicación personal, 15 de mayo de 2019).

Las estudiantes sí se habían acercado a las autoridades del campus, al Director de División, al Secretario Académico, y no habían recibido la respuesta que ellas esperaban (Autoridades 1, comunicación personal, 19 de mayo de 2019).

Sin embargo, las autoridades también argumentan que el caso Kala fue una oportunidad de aprendizaje para actuar, de tal manera que con ello se "había creado una institucionalidad" (Autoridades 2, comunicación personal, 15 de mayo de 2019). Así, a raíz de estos sucesos, se elaboró un protocolo y se creó la Unidad de Género (conocida como UGénero), la entidad que sería responsable de dar seguimiento a las denuncias por violencia de género. Asimismo, al año siguiente (2016) las autoridades de la universidad organizaron la realización de un Taller de Igualdad de Género, al que asistieron 13 directivos, con el fin de "definir los criterios para impulsar una cultura organizacional con perspectiva de género y derechos humanos en la Universidad de Guanajuato (UG), así como profundizar sus conocimientos en el tema" ${ }^{\circ}$. De hecho, para algunas de las autoridades de la universidad, el tema ya se estaba abordando antes de que estallara el caso:

Pues entendemos que aquí el tema ha sido abordado, es parte de lo que aquí se discute. Obviamente, también todo lo que tiene que ver con las tendencias, principalmente con el ambiente, perspectiva de género, desigualdades, son parte de lo que nosotros cotidianamente discutimos a la larga, y formamos a

\footnotetext{
${ }^{8}$ La nota sobre este taller puede encontrarse en http://www.dcv.ugto.mx/ugenero/noticias/13-directivos-de-laug-asisten-a-taller-de-igualdad-de-genero?fbclid=IwAR3GG6jJjexB3QH6oykX3UvRlbUh4FvMpPRXTtdHNGPUjgQgDmjEVgm6NA
} 
nuestros alumnos" (Autoridades 2, comunicación personal, 15 de mayo de 2019).

Sin embargo, para algunos y algunas docentes, esto se trataba más bien de una simulación, pues, más allá del discurso, no había espacios que permitieran realmente formarse en temas de género, paso fundamental para una transversalización de la perspectiva de género:

No había prácticamente nada. Se daba por sentado que era un tema importante, un tema interesante, pero no había ni las instancias académicas ni los lugares académicos dónde dirimir, dónde aprender, dónde enseñar y, dónde educar temas de género (Docentes 1, comunicación personal, 15 de mayo de 2019).

El protocolo, la unidad de género y los talleres no parecieron ser suficientes, como se puso de manifiesto dos años después, cuando estallaron los sucesos de la Colectiva Alumnas UG en Sororidad. Como apuntó una de las autoridades entrevistadas, "a través de una denuncia pública esta Colectiva nos deja saber que hay casos que no se mueven, que hay casos que eran del conocimiento de autoridades y que no hicieron nada" (Autoridades 1, comunicación personal, 17 de mayo de 2019).

\section{La Colectiva de Alumnas UG en Sororidad y las denuncias de acoso}

La vida de la Universidad Autónoma de Guanajuato, y concretamente del campus León, se vio alterada el 14 de agosto de 2018, cuando un grupo de estudiantes ofreció una rueda de prensa en el patio de la universidad, donde declaró públicamente que existen casos de acoso por parte de profesores de la universidad. Varias cuestiones fueron decisivas para entender lo que ocurriría posteriormente.

En primer lugar, todavía en ese momento no había ninguna queja formal interpuesta en las instancias correspondientes (UGénero); es más, los profesores acusados apuntaron que tardarían más de dos meses (hasta el $1^{\circ}$ de noviembre) para saber quién y por qué se les acusaba. 
La Colectiva y la Asociación Civil Las Libres, por su parte, argumentarían que en realidad la queja ya estaba ahí, puesto que una profesora, como tutora de algunas estudiantes, se había acercado a las autoridades para exponer su preocupación por la reiteración de conductas (de los profesores) que podían ser catalogadas como violencia de género. Las autoridades corroboraron este hecho, de que sí se había producido este acercamiento con la profesora, pero, por diferentes circunstancias, ya no se investigó nada más. De acuerdo con Las Libres, es obligación de la institución iniciar una investigación en el momento en que tienen conocimiento de alguna posible conducta indebida, pero en este caso las autoridades fueron omisas: "las chicas ya habían llegado con diversas autoridades. Pero nadie había activado nada" (Colectiva 4, comunicación personal, 16 de mayo de 2019).

La nula respuesta recibida por parte de las autoridades hizo que la profesora se acercara a un medio de comunicación (Zona Franca) que había tenido una agenda muy explícita en temas de género. Es ahí cuando se incorpora Las Libres, y desde ese momento la asociación asume el papel de "ayudante", acompañando y asesorando a las estudiantes en su proceso de denuncia. Al mismo tiempo, lo que hasta entonces había sido un grupo de estudiantes que se habían ido juntando a partir del reconocimiento de una problemática compartida, deciden conformarse como la Colectiva Alumnas UG en Sororidad.

La falta de respuestas por parte de las autoridades y la desconfianza provocó que se buscaran vías alternativas de intervención, pues se consideraba que recurrir a la denuncia formal dentro de la universidad era condenar el caso al cajón del olvido, al silencio. Era necesario visibilizar lo que estaba ocurriendo en la universidad antes de que se abriera el caso en la instancia formal. Esta exposición pública tendría una importancia decisiva en el proceso, puesto que la presión externa impidió que se siguiera manteniendo la táctica de la negación y la dilación: "lo que me ha tocado vivir a mí es una respuesta muy reactiva, presionada por la opinión pública, lo cual implica que desde mi punto de vista procesos, protocolos y plazos se han hecho a la carrera" (Denunciados 1, comunicación personal, 15 de mayo de 2019).

La rueda de prensa, entonces, fue una estrategia deliberada que dio un campo de acción mucho más amplio a las denunciantes, puesto que al evitar en un primer momento las vías 
formales, no se quedaba circunscrito a las reglas establecidas en este ámbito, lo cual permitió, entre otras cosas, que se pudieran hacer públicos los nombres de algunos de los profesores que estaban siendo acusados, que, como se verá más adelante, fue crucial para el desarrollo de los acontecimientos.

Otro punto decisivo fue la elección de la fecha en la que se ofreció la rueda de prensa: un día antes del informe del rector, factor de atracción para los medios que fueron convocados. No debe olvidarse que se estaba a menos de un año de la posible ratificación del rector, por lo que no iba a pasar desapercibida una noticia que señalaba de forma explícita a la universidad como un espacio que no estaba libre de violencias, justo a un día de que la máxima autoridad presentara su informe de actividades.

La rueda de prensa tuvo una buena concurrencia de medios y una cobertura que incluso trascendió las propias fronteras del estado. Además, se aprovechó para hacer un llamado específico al rector para que, al día siguiente, durante su informe, diera respuesta a la demanda de castigo a los culpables y revisión de los procedimientos para asegurarse que organismos como UGénero fueran realmente efectivos (Zona Franca, 2018). Es una interpelación directa a la máxima autoridad, frente a los medios, y con una queja explícita de que la institución no sólo no ha respondido ante los casos de acoso, sino que ha sido cómplice, al proteger a quienes cometen este tipo de faltas:

La opción que estaba, que fue la rueda de prensa, era eso, el decir ya nos tienen hartas aunque no me haya pasado a mí, aunque no haya ido a denunciar a UGénero, pero ya estamos hartas de que se hayan quedado en chismes de pasillo; porque sí eran chismes de pasillo, pero no porque no fueran verdad, sino porque no había dónde acudir o dónde decirlo, y menos a ellos: "oye, tu amigo me está acosando. No lo iban a creer en nada y eso fue lo que nos motivó a hacer la rueda de prensa" (Colectiva 2, comunicación personal, 16 de mayo de 2019). 
Tenía que ser algo que les doliera en el prestigio de la Universidad para que nos hicieran caso, entonces como lo hicimos fue la única forma para que hubiera este resultado (Colectiva 3, comunicación personal, 17 de mayo de 2019).

Un último punto que vale la pena destacar es el formato de la rueda de prensa y algunos de sus contenidos más importantes. En primer lugar, tras una breve presentación de Verónica Cruz, de Las Libres, la parte central es la lectura de un texto por parte de estudiantes de la UG, en donde se señala que la universidad no es un espacio seguro para las mujeres. En el cierre, dos profesoras toman la palabra (una, denunciando su propio caso de hostigamiento, y la otra, apoyando a la Colectiva) y Verónica Cruz responde a las preguntas puntuales de los medios. En todo momento se plantea como una denuncia colectiva, pues no se quiere aludir al caso específico de una alumna determinada, sino que se busca que la atención se centre en el acosador, y no en la víctima. Esto es un tema fundamental que se mantuvo durante todo el proceso: en ningún momento se sabe quiénes son las denunciantes; cuando las estudiantes hablan, lo hacen como parte de una Colectiva, no como la víctima específica. Ello permitió centrar la atención en los agresores, y evitó cualquier discusión que pudiera implicar algún tipo de responsabilidad por parte de las agraviadas.

En la misma rueda de prensa, las estudiantes decidieron hacer públicos los nombres de cinco de los profesores implicados en los 17 casos que se presentaron (que acabaron siendo 14). Dos razones explican esta decisión, que fue muy cuestionada especialmente por los profesores involucrados, por un lado, el impacto mediático que tendría, pues no es lo mismo hablar de acoso en la universidad, de manera genérica, que poner nombres y apellidos; por otro lado, se buscaba reforzar esa idea de que la atención debía centrarse en los acosadores y no en las víctimas. "Era que la atención ya no se volcara en nosotras, porque nosotras íbamos a dar la cara y que no nos señalaran, sino que fuera directamente a ellos, y eso fue lo que nos motivó" (Colectiva 2, comunicación personal, 16 de mayo de 2019).

Esto tuvo un alto costo para los profesores señalados, por las implicaciones tanto para ellos, como para sus familias. Fue el aspecto que más los incomodó, además porque en su caso 
fueron señalados sin saber específicamente quién ni por qué los estaban acusando. Recordemos que cuando las estudiantes deciden ofrecer la rueda de prensa, en realidad no había ningún expediente abierto, y aunque la Colectiva y Las Libres señalan que las autoridades ya tenían conocimiento de los casos, éste no se da a través de un mecanismo formal institucional, de manera que cuando los profesores acudieron con las autoridades para saber de qué se les acusaba, argumentan que nadie pudo decirles.

La estrategia de exponer a los profesores atrajo la atención, tanto de medios de comunicación como de las propias autoridades, cuya reacción fue inmediata. Poco tiempo después de la rueda, el rector busca a las estudiantes, y celebran una primera reunión, en donde se les pide que presenten las denuncias de manera formal, para que la institución pueda actuar. Se da un estira y afloja — una negociación donde las partes van cediendo_-, pues las denunciantes querían mantenerlo como una denuncia colectiva, pero el reglamento y el protocolo obligaba a que la solicitud de atención debía ser presentada por la persona en específico:

Lo primero que entregó Verónica Cruz después de la denuncia pública fueron párrafos de testimonios sin nombre, sin fecha, sin nada. Con eso, ¿Qué era posible hacer? Lo que se hizo fue tratar de ganar la confianza de las denunciantes para que denunciaran (Autoridades 1, comunicación personal, 17 de mayo de 2019).

Eso explica la tardanza para que los profesores conocieran las denuncias, y pudieran dar respuesta a las mismas. Los tiempos, además, se alargaron porque las actividades de la Comisión se tuvieron que interrumpir para poder capacitar a las personas que la conformaban, pues una de las cuestiones que surgieron es que dichas personas no conocían la problemática de género e incurrieron en una serie de faltas de procedimiento que llevaron a procesos de revictimización.

Finalmente, el 29 de enero de 2019 se dio a conocer la resolución de la comisión: de los catorce casos de acoso que se habían presentado, a seis profesores se les aplicó una suspensión de labores de ocho días (que era la sanción máxima que podían recibir), tras 
acreditar su responsabilidad en los hechos; tres de ellos recibieron una amonestación, dos fueron absueltos, y en tres casos no hubo elementos suficientes para dar una resolución. ¿Qué ocurrió durante estos meses? ¿De qué manera la universidad atendió el caso? En el siguiente apartado analizaremos los elementos más importantes del proceso, de manera que podamos dar respuesta a las interrogantes.

\section{La Universidad de Guanajuato frente a las denuncias de acoso}

Como se señaló, la denuncia contra el profesor Julio César Kala (2015) había provocado que la institución reaccionara, actuando tanto en el ámbito normativo (diseño y aprobación del protocolo en 2017), como en el ámbito de la prevención (talleres y cursos). Sin embargo, cuando surge el caso de la Colectiva Alumnas UG en Sororidad se puso en evidencia que estas medidas no habían sido suficientes y que se estaba muy lejos de atender de manera correcta la problemática de la violencia de género. El descontento por parte de todas las partes (estudiantes que pusieron la queja, profesores acusados y la comunidad universitaria en general) es una muestra de ello, pues todo el mundo quedó con la sensación de que no se había hecho justicia y de que la universidad no estaba respondiendo adecuadamente. En este apartado analizaremos las principales fallas que se presentaron, con el fin de identificar cómo mejorar los procesos.

El primer aspecto que hay que considerar es el normativo. Si bien es cierto que la UG había aprobado en octubre de 2017 un protocolo para la atención de la violencia de género en la universidad, éste resultó insuficiente. Al revisar su contenido, se constata que se centra más en las conductas que deben emplearse durante el proceso que en el establecimiento de los pasos concretos, en el señalamiento de las facultades específicas de las diferentes instancias que intervienen, y de los plazos y tiempos en los que deben actuar. Una de las autoridades entrevistadas, reconoció las propias limitaciones del instrumento, al señalar que "es una institucionalidad limitada, por lo que alcanzo a ver, porque realmente no crea una estructura del procesamiento, sino básicamente de acompañamiento. Si acaso de canalización” (Autoridades 2, comunicación personal, 15 de mayo de 2019). 
Así, el protocolo determina que, tras recibir una solicitud de atención, tendrá lugar una primera cita, para posteriormente establecer un Plan de Atención y un Plan de Seguridad; existe claridad con respecto a la atención psicológica o jurídica, y también en cuanto a las medidas que puedan contribuir a asegurar el entorno de la víctima. Sin embargo, es muy poco preciso sobre cómo y quién debe realizar la investigación o sobre las posibles sanciones que se pueden aplicar.

Un primer malentendido se produjo a raíz de la falta de precisión con respecto a la manera en que se abre un expediente, y el papel otorgado a las autoridades de la universidad. En la Guía Básica que se presenta en el portal de la universidad, se establece que cuando se identifique alguna forma de violencia, se debe contactar a "alguna persona, ya sea del personal académico o administrativo, personal de apoyo o una autoridad, para que te brinde orientación", aun cuando a continuación se señala que la "Ventanilla UGénero es la instancia especializada para brindarte apoyo y en la cual, si así es tu voluntad, puedes reportar la situación" (Universidad de Guanajuato, s.f.). El artículo VII.1 del Protocolo de Atención a Casos de Violencia de Género de la Universidad de Guanajuato establece que:

La solicitud de atención deberá ser presentada directamente por la persona que manifiesta ser afectada. En el caso de que una tercera persona tenga conocimiento de algún hecho de violencia de género deberá hacer de conocimiento a la Ventanilla UGénero, para dar seguimiento a la solicitud de atención directamente con la persona afectada (Universidad de Guanajuato, 2017).

Sin embargo, el personal de UGénero argumenta que el caso nunca llegó a la Ventanilla, sino que el rector se hizo cargo personalmente, aun cuando el reglamento establece que la atención siempre debe ser a través de la Unidad de Género. La falta de respuestas por parte de las autoridades más cercanas a las estudiantes, la desconfianza hacia dichas autoridades y el temor a las "tradiciones de lealtad y secreto" que menciona Barreto (2017) para explicar las formas de negación institucional, hizo que las estudiantes buscaran interlocutores más estratégicos: "las chicas, con toda la razón, fueron a pedir a la autoridad directa el apoyo y 
siempre es negado, entonces, claro, su último recurso es llegar a la puerta más grande" (Autoridades 3, comunicación personal, 17 de mayo de 2019). El rector, ante la presión social y mediática, decide atenderlas personalmente. El problema es que eso generó confusión entre las mismas autoridades y malestar en los profesores acusados, que consideraban que el reglamento no se estaba aplicando adecuadamente.

Cuando tiene lugar la primera reunión con el rector, y se les pide la presentación de los casos específicos, esto no se hace. Las estudiantes, querían mantener la denuncia como una denuncia colectiva, anónima, lo cual contravenía lo establecido por el protocolo. Transcurrieron varias semanas hasta que se presentaron los casos. Mientras tanto, como se mencionó, los profesores implicados, expuestos públicamente, sentían que las autoridades no habían reaccionado de la manera adecuada, habían sido condescendientes con las fechas, dejándoles desprotegidos, y sin la posibilidad de conocer su caso: "siempre la soga se parte por el lado más débil, y el lado más débil en este caso somos los profesores, ¿los profesores, por qué?, porque no tenemos el respaldo de la opinión pública, porque no se ha escuchado nuestra voz" (Denunciados 1, comunicación personal, 15 de mayo de 2019).

Una vez aclarado y abierto el expediente, surgió un nuevo problema con los procedimientos. En el título XI se habla de la "canalización del caso a las instancias universitarias competentes para conocer del mismo", y en el título XII se señala que, "dichas instancias, dado el caso, impondrán las sanciones que correspondan de acuerdo con el marco normativo y en consideración a la resolución emitida por la autoridad competente" (Universidad de Guanajuato, 2017). Estas instancias competentes son las Comisiones de Honor y Justicia de los Órganos Académicos Colegiados, la Contraloría General de la Universidad y la Dirección de Recursos Humanos. Aquí nos encontramos con una nueva problemática, especialmente cuando las personas agresoras pertenecen a la comunidad universitaria, y es que las Comisiones de Honor y Justicia carecen del principio de imparcialidad que se establece en el propio protocolo, dado que, en muchas ocasiones, están conformadas por colegas y amigos de las personas involucradas, lo cual impedía que se pudiera actuar de manera justa e imparcial: "las Comisiones de Honor y Justicia, que son las que tradicionalmente podrían 
tratar estos temas, se muestran insuficientes o no aseguran imparcialidad o no aseguran un tratamiento adecuado" (Docentes 1, comunicación personal, 15 de mayo de 2019).

El impacto mediático del caso provocó que el rector girara instrucciones para que en esta ocasión fuera la Comisión de Honor y Justicia del Consejo General quien acabara emitiendo una resolución al respecto.

Una vez que se estableció quién resolvería el caso, tuvieron lugar las primeras audiencias con estudiantes, lo cual llevó a que se incurriera en una serie de errores que hicieron que las víctimas se sintieran maltratadas, con procesos de revictimización fuertes: fueron entrevistas poco cuidadas con respecto a las personas que participaron en las mismas, el tipo de preguntas que se hicieron y la manera cómo se cuestionaba. Quienes iban a tomar la decisión evidenciaron carecer de las bases necesarias para comprender cómo atender estos casos con perspectiva de género. Éste es uno de los grandes retos que enfrentan las universidades frente a la violencia de género: si bien puede existir la voluntad de atender el problema, no existe una preparación suficiente para hacerlo.

Este hecho provocó que las estudiantes, acompañadas por Las Libres, acudieran a la Procuraduría de Derechos Humanos del Estado de Guanajuato. La recomendación de esta instancia fue contundente: antes de seguir adelante, era necesario capacitar a las personas, lo cual provocó un retraso aún mayor, con el consiguiente malestar de los profesores acusados. Lo que se puso de manifiesto durante todo el proceso tiene que ver fundamentalmente con aspectos culturales. Un tema muy importante es la falta de sensibilización, en realidad no se acaba de comprender lo que hay detrás de ese gran paraguas que es la violencia de género. Esto se refleja en la normalización de ciertas formas de comportamiento ${ }^{9}$, que incluso cuando tienen una carga de violencia, no son vistas como tales, sino, quizá, como algunos errores menores: "Es un problema extendido. Es un problema, sin temor a equivocarme,

\footnotetext{
${ }^{9}$ Se usa el concepto de normalización de la violencia en el sentido de Evangelista (2019), quien señala que este concepto "obliga a referirse a conceptualizaciones de ésta que van más allá de la violencia directa y visible de carácter relacional y que claramente se materializa en actos físicos y psicológicos delimitados en el tiempo y el espacio (...) y ocuparse de los actos invisibles que se expresan a través de la subordinación a patrones culturales y económicos impuestos por grupos de poder y hegemónicos con efectos incluso más graves que los que ocasiona la violencia física" (Evangelista, 2019, p. 87).
} 
subestimado, que no se entiende. 'Pues es que traía esa falda, se sienta así'; hay un desconocimiento impresionante, entonces se ve como natural pues siempre ha pasado" (Autoridades 3, comunicación personal, 17 de mayo de 2019).

Esto se ve muy claramente cuando se platica con los profesores que fueron acusados por las estudiantes. No se entiende la violencia — las violencias - como un fenómeno con múltiples aristas y muy diferentes formas de manifestarse, que van desde comentarios, chistes con contenidos sexista, formas de aproximarse y comunicarse con las personas, hasta expresiones que implican un mayor nivel de agresión ${ }^{10}$. Por el contrario, se sienten injustamente tratados, porque consideran que no deben ser encasillados como si fueran violadores:

En el imaginario para un sector es un depredador sexual, ¿qué hace un depredador sexual? ¿Violaste, la tocaste, la forzaste? o sea no te cabe en la cabeza que si alguien te dijo "guapa", o que alguien te dijo, "oye, qué bonita sonrisa tienes” o “¿por qué te ríes?” no, en lo imaginario es "las tocaron”, "las forzaron", entonces eso no lo dice la universidad, eso se resuelve en una procuraduría.

El propio discurso de la universidad las ha apoyado, en el sentido que las autoridades que dicen, "si se percibe, es" entonces ya no tienes nada qué hacer (Denunciados 1, comunicación personal, 15 de mayo de 2019).

No obstante, sí hubo entre algunos de ellos el reconocimiento de que se había actuado mal: "no puedo decir 'no hice nada', sí hice y es lo que asumo" (Denunciados 2, comunicación personal, 16 de mayo de 2019). Pero en general se observa una resistencia a ver como parte de un mismo problema esas diferentes formas de generar violencia, y se considera que son cuestiones distintas que deben ser abordadas de manera diferenciada. Es importante tener en cuenta que muchas de las expresiones de la violencia de género que se viven pueden ser manifestaciones que entrarían más en la categoría de "micromachismos" (de acuerdo con la definición de Bonino, 2005). El problema es que quien incurre en este tipo de prácticas no

10 Violencia "como un continnum, desde niveles macroestructurales hasta microscópicos, desde manifestaciones físicas y corporales hasta simbólicas, desde episodios extraordinarios hasta cotidianos y desde situaciones graves hasta leves" (Evangelista, 2019, p. 87). 
las identifica como otra forma de ejercer violencia, que parte de una relación desigual, que genera abusos de poder. Al no verlas así, se sienten injustamente tratados, y reaccionan a la defensiva, pero estableciendo contundentemente que ellos no son depredadores sexuales, y que sienten que los tratan como si fueran violadores o feminicidas. De esta manera, las personas acusadas de haber generado situaciones de violencia comienzan a construir un discurso mediante el cual buscan ser vistos como víctimas del sistema: reconocen que hay violencia de género en la sociedad, reconocen que es una problemática para considerar, pero, en general, no se ven como parte del sistema, sino que consideran que han sido una suerte de chivos expiatorios.

A mí lo que me queda es eso, el clima laboral que se recomponga (porque no se ha recompuesto), la universidad que opere, no por quedar bien con la opinión pública, sino por eliminar estas situaciones, y que haya un proceso, ya restaurativo, y de platicar todo esto, y de evitar conductas, y de capacitación; pero no como una respuesta a la presión mediática, sino como un compromiso real, donde además uno mismo pueda decir, no espérate, yo también fui víctima, o sea yo he sido víctima de esto, no me conviertas en sólo lobo, lobo, lobo, sino que yo soy víctima, o sea revisémonos todos, qué estamos haciendo (Denunciados 1, comunicación personal, 15 de mayo de 2019).

En algunos casos, no distinguen entre la vía penal para atender un caso de violencia de género, y los mecanismos institucionales que una organización puede desarrollar para garantizar un espacio libre de violencia. Algunas de las personas entrevistadas señalaron que no entendían por qué las estudiantes no habían acudido a la vía penal, si tan grave era el asunto, sin comprender que son dos ámbitos diferentes de acción e intervención.

Decíamos "vámonos mejor al Ministerio Público, o que las chicas se vayan a Ministerio Público” y decía el abogado “¿pero por qué ahí?” pues porque ahí por lo menos tienen más experiencia que la universidad en manejo de denuncias, a eso se dedican, y que estamos ciertos que tal vez errores sí se han 
cometido, pero no a un nivel tan grave como al menos parecía en la rueda de prensa (Denunciados 2, comunicación personal, 16 de mayo de 2019).

Esto no quedó nada más en un nivel individual o personal, sino que se puso de manifiesto de cara a la comunidad, a través de la aparición de una serie de carteles en apoyo a los profesores acusados y acusando a su vez de acoso a quienes respaldaban la demanda, lo cual provocó que se diera la situación de "alumnas violentadas por sus propios compañeros" (Docentes 5, comunicación personal, 15 de mayo de 2019), y de divisiones en la comunidad en general:

[Enfrentamientos] nunca directamente a nosotras de parte de compañeros nunca, comenzamos pegas entre compañeros y ponían otra pega abajo y ponían "yo no te creo" o "yo sí te creo, tal profesor acosador". Me daban mucha pena las estudiantes defendiendo, porque también las afecta a ellas (Colectiva 3, comunicación personal, 17 de mayo de 2019).

De esta manera, el ambiente en la universidad se fue enrareciendo cada vez más. Las estudiantes, y las profesoras involucradas, señalan que recibieron todo tipo de amenazas, y que se propició un ambiente de rechazo hacia ellas. De la misma manera, los profesores señalados mantuvieron ese discurso de victimización, resultado de no haber entendido el fenómeno de la violencia de género. Para cuando se anunció la sanción que recibieron algunos de los profesores, la fractura ya estaba dada, y el descontento era generalizado entre todas las personas involucradas en el caso. La Colectiva se quedó con la sensación de que nunca hubo un verdadero interés por parte de las autoridades para ir al fondo del problema de la violencia de género:

En realidad, no hubo nunca un interés ni preocupación ni una reflexión de lo que estaba pasando, y yo incluso creo que sigue sin haberla, ni la van a tener. (...) Entonces, hubo mucho desencanto, si la relación con las autoridades ya estaba fragmentada ya había una falta de confianza hacia las autoridades, esto terminó por generar una ruptura completamente (Colectiva 1, comunicación personal, 16 de mayo de 2019). 
Lo que es peor, para muchos quedó la sensación de que como los implicados eran amigos de las autoridades, más allá de la sanción de los ocho días, en el interior de la universidad siguen teniendo una situación de privilegio: “¿Qué ha hecho la institución? Los ha ido protegiendo. Si tú te fijas en la lista de las personas que han recibido apoyos últimamente para su investigación científica, están estos. O sea, ya los lavaron" (Docentes 4, comunicación personal, 17 de mayo de 2019).

Por otro lado, como se ha mencionado, los profesores que fueron expuestos públicamente resintieron la falta de respaldo de la institución, que actuó fundamentalmente por las presiones que estaba recibiendo por el impacto mediático del caso:

Porque tú confías en la institución, y no porque la institución me apañe, sino porque sea imparcial, porque responda de manera justa, y responda a un problema real que está jodiendo mujeres en este país, pero no tratando, en este caso, de quedar bien con la opinión pública. (Denunciados 1, comunicación personal, 15 de mayo de 2019).

Con la universidad estoy muy enojado y muy decepcionado, mi enojo es con ellos y no con la universidad como institución, sino con los hombres que manejan la institución actualmente, que no quisieron reconocer que no sabían cómo hacerlo y entonces lo fácil fue tomarnos como chivos expiatorios; volviendo a aclarar que no estoy diciendo que soy $100 \%$ inocente, pero las cosas había que plantearlas como eran, y no decir "resolvemos cortando por lo delgado", eso sí me pareció lamentable (Denunciados 2, comunicación personal, 16 de mayo de 2019).

Las autoridades son conscientes de la fractura del clima laboral, apuntan que "la gente está muy enojada" y que "hay mucha intolerancia de todas las partes" (Autoridades 3, comunicación persona, 17 de mayo de 2019), y esto también lo resiente el profesorado en general, que percibe cómo está afectando a la vida cotidiana en la universidad: 
Me apena muchísimo, porque tienen un mal ambiente laboral..., porque no nada más son las personas involucradas en el caso, sino son todas. (...) Las reuniones son tensas, las actividades son no sólo tensas, sino que ya no va casi nadie. No festejar un día del maestro porque no hay armonía (Docentes 2, comunicación personal, 15 de mayo de 2019).

Los mecanismos institucionales resultaron insuficientes para atender la problemática de la violencia de género; al final, todo el mundo se quedó con la sensación de que se estaba igual o peor que antes: las estudiantes no sienten que recibieron una reparación adecuada, además de que no tienen ninguna garantía de que hechos así no se vuelvan a repetir; los profesores se sintieron maltratados y expuestos por una institución que, según ellos, actuó presionada por la opinión pública, en lugar de respetar los procedimientos formales. En el siguiente apartado, se formularán las hipótesis derivadas de la investigación, que nos permitan esbozar, de acuerdo con lo que establece la teoría fundamentada, la teoría sustantiva en torno a los problemas que enfrentan las universidades para atender la problemática de la violencia de género.

\section{Reflexiones finales}

En una investigación previa (Varela, 2020), se hizo hincapié en que las universidades son espacios "generizados", en donde, como señala Cerva, se "producen y reproducen relaciones de poder que tienen un efecto en la desigualdad de género" (Cerva, 2017, p.21). Acker, ya en los años noventa, apuntaba que en las organizaciones se tendía a invisibilizar el impacto diferenciado que tiene, en términos de ventajas y desventajas, el hecho de ser hombre o mujer, y, en cambio, se imponía un discurso que tiende a conceptualizarlas como neutrales en términos de género (Acker, 1990, p.142). Este aspecto es fundamental para entender cómo se posiciona cada quien ante fenómenos como el de la violencia de género, pues hay quienes lo ven simplemente como hechos aislados, y coyunturales, y quienes sí identifican una serie

de dinámicas de carácter estructural que exponen las relaciones asimétricas entre hombres y mujeres. 
A lo largo de estas páginas, se ha mostrado el proceso institucional que se desencadenó a partir del momento en que una colectiva de estudiantes de la UG decide denunciar públicamente las situaciones de acoso que se viven en la institución. Dadas las características del caso y la poca información que se tiene sobre el tema, se optó por dar prioridad a las voces que participaron en el proceso. Se trataba de ir construyendo una serie de hipótesis a partir de los hallazgos obtenidos durante la investigación, que a continuación se presentan. Primero, el carácter conservador de la sociedad guanajuatense no ayudó a impulsar cambios más de fondo, pero sería importante revisar cómo actúan otras universidades, con el fin de determinar hasta qué punto es generalizable esta forma de comportamiento. En principio, hay indicios para considerar que universidades en ambientes conservadores son más propensas a adoptar la estrategia de negación y silenciamiento a la que aluden tanto Barreto, como Mingo y Moreno, porque desde una posición conservadora hay una resistencia a ver la violencia de género como un problema estructural que requeriría transformaciones de fondo de la sociedad.

Segundo, es importante contar con una normatividad específica, con instrumentos precisos y certeros sobre cómo actuar cuando se presenten casos de violencia de género. Si los protocolos no cuentan con la información suficiente, se amplía el margen para la discrecionalidad, provocando incertidumbre e inseguridad entre las personas involucradas. Reglas claras que son respetadas propician un ambiente más seguro, y por tanto facilitan los procesos de reparación frente al daño ocasionado y, sobre todo, la garantía para la no repetición de los hechos.

Tercero, un tema clave para identificar los problemas suscitados durante el proceso tiene que ver con el modo en que la universidad actúa frente a la problemática de la violencia de género: tanto en el caso Kala como en el de la Colectiva, las autoridades intervienen de manera reactiva, cuando ya se han visto en una situación límite de tensión que no les deja otra opción, pero al hacerlo bajo una situación de presión su actuar es visto como errático, poco comprometido, y más cercano a los juegos de simulación (a los que alude Phips) que a un verdadero interés por acabar con el problema. Las decisiones son, entonces, precipitadas, sin que exista una idea clara de qué es lo que conviene hacer: "Las universidades han sido muy 
reactivas, han estado muy presionadas por la opinión pública por decir y demostrar tenemos un protocolo, estamos actuando" (Denunciados 1, comunicación personal, 15 de mayo de 2019). Una hipótesis surgida de la investigación es que, en realidad, el problema de la violencia de género sigue sin ser entendido: se confunde lo que es, y al tener ciertas prácticas tan normalizadas, no se alcanza a vislumbrar su impacto sobre las relaciones sociales. El no conocer la problemática, el no tener claridad sobre contra qué se está actuando, ni cuáles son los factores que influyen, hace que el tema no sea abordado de manera estructural, sino como reacción ante situaciones específicas. Esto hace que en realidad no quede claro cuál ha sido el rol de las autoridades frente a la denuncia de La Colectiva: para los profesores acusados, la universidad se puso del lado de las estudiantes, sin apoyarlos y permitiendo que el reglamento no se respetara; en cambio, quienes presentaron las denuncias se quedaron con la sensación de que las autoridades habían actuado como verdaderos opositores a su causa, simulando un interés por enfrentar el problema, cuando en realidad solaparon a los docentes acusados. Prueba de ello es que no hay una disposición real a bajar la discusión para que permee en toda la comunidad universitaria (la falta de capacitación del comité que debía investigar y deliberar sobre el caso, es una muestra clara de ella, como lo es el hecho de que no haya una discusión abierta sobre lo que está pasando en la institución, sino que se prefiere "barrer bajo la alfombra”).

Cuarto, en ese sentido, cabría pensar que la estructura vertical de la institución que se señaló al inicio de este trabajo, podría ser un factor que coadyuvara a propiciar ciertas prácticas y a generar procesos de reflexión sobre cómo se viven éstas en la universidad; sin embargo, nunca se dieron estos procesos de diálogo hacia abajo, de tal manera que lo que señalaban las máximas autoridades quedaron en simples declaraciones de buenas intenciones, que eran vistas más como reacciones políticamente correctas, que como una verdadera voluntad de incidir sobre la comunidad:

El discurso de siempre "estamos hablando con los Directores de Divisiones" o peor, "con los Rectores de Campus para que de la Rectoría de Campus baje", todo lo que había dicho el Secretario General no baja operativamente (Docentes 6, comunicación personal, 17 de mayo de 2019). 
Pero el trabajo real de ir a los salones, de sensibilizar, de exigir a las autoridades que hagan el trabajo, eso no sucede (Docentes 6, comunicación personal, 17 de mayo de 2019).

Quinto, la investigación puso de relieve la importancia que pueden tener los grupos de poder dentro de la universidad, especialmente cuando alguno de sus integrantes es acusado de haber cometido un acto de violencia de género, y el resto de colegas tiende a desacreditar a quienes realizan la acusación y a defender al supuesto agresor. En la medida en que estos grupos tengan más capacidad de incidencia, es más probable que nos encontremos con universidades que "obstaculizan sistemáticamente la denuncia e imponen de mil maneras el silencio como forma de no reconocer la violencia de género que ahí sucede” (Evangelista, 2017, p. 338).

Sexto, al actuar reactivamente, los esfuerzos se han centrado en atender los casos de violencia de género que se presentan, en lugar de atender todo lo que tiene que ver con la prevención: "No hay un tema de prevención de ningún sentido y de conciencia de que son cosas que pasan, aunque no se hablen" (Docentes 2, comunicación personal, 15 de mayo de 2019). Al reducir al mínimo las actividades encaminadas a la prevención de la violencia, ésta seguirá estando presente; puede que mejoren los mecanismos para atenderla, pero no se reducirá, mientras no se hagan esfuerzos reales por erradicar la violencia.

Séptimo, como ya se ha destacado en otras investigaciones (Barreto, 2017; Barreto 2018; Phips, 2018), las movilizaciones llevadas a cabo por las estudiantes han sido fundamentales para romper con el silencio institucional en las universidades. Cuando las estudiantes han sido capaces de alzar sus voces, de atraer la atención de la opinión pública, es cuando se ha logrado visibilizar la problemática y hacer que las autoridades no puedan seguir comportándose como si no supieran de ella.

En conclusión, lo que se ha reflejado a través de los testimonios de las diferentes personas, es que en realidad no se ha dado una verdadera transversalización de la perspectiva de 
género $^{11}$. Para el caso de la violencia de género, esto implica entender que los casos de violencia no son hechos aislados, sino que responden a una problemática más compleja, derivada de la manera en que las relaciones entre hombres y mujeres responden a formas de dominación que generan desequilibrios en las mismas. Se trata de ir más allá de contar con una normatividad adecuada o de asegurarse que ésta se cumpla adecuadamente; se trata de entender lo que hay detrás de la violencia, para poder atajarla, de aceptar que ello implica ir a cuestiones más estructurales y que tienen que ver también con los valores y las conductas de la sociedad:

Las instituciones tienen que dejar la cultura de la simulación, eso en términos generales, es decir, si tienen protocolos tiene que aplicarlos, pero más que aplicarlos, tienen que hacer un proceso de educación en temas jurídicos, de educación en cultura de género, de educación en cultura de paz, de masculinidades (Denunciados 1, comunicación personal, 15 de mayo de 2019). Y en ese sentido es la sensibilización, es decir, tenemos que estar y eso se ve a diario porque es un tema como educación o valores o todo eso es a largo plazo, pero hay que empezar y tiene que estar, pues en todo, que se materialice en todo, desde los planes, se materialice en los recursos que se destinan, o sea, en acciones concretas y palpables (Autoridades 2, comunicación personal, 15 de mayo de 2019).

Esto tiene que madurar desde dentro tiene que crecer al interior de las instituciones universitarias y tiene que ir poco a poco permeando por todas partes, tiene que ser invasivo, como un virus que entra y que va extendiéndose y que va haciendo que haya una cultura de género en todas partes (Docentes 1, comunicación personal, 15 de mayo de 2019).

El caso abierto por la Colectiva sirvió para alertar sobre lo que está ocurriendo en las universidades. Son muchas las lecciones que se pueden extraer, y permitirá tomar decisiones

11 Rodríguez Gustá (2008) identifica cuatro categorías diferentes de políticas que hacen frente a las desigualdades: las de acción afirmativa, políticas para las mujeres, con perspectiva de género y las de transversalización de género, que implica un abordaje sistematizado e integral. 
para mejorar las respuestas de las instituciones de educación superior frente a la violencia de género. Es largo el camino por recorrer, pero el caso nos permitió identificar por dónde debe ir dicho camino:

Ellas hicieron lo que pudieron para llamar la atención y la verdad es que hay que estarles profundamente agradecidas de haber puesto el dedo en la llaga, una llaga que duele, una llaga que lastima, pero hay que ponerla lamentablemente, y que yo no sé si es por omisión, por temor, no me importa, pero ellas lo ponen (Autoridades 3, comunicación personal, 17 de mayo de 2019).

\section{Referencias bibliográficas}

Acker, Joan. (1990). Hierarchies, Jobs, Bodies: a Theory of Gendered Organizations. Gender and Society, 4(2), 139-158.

Aguilar, Rubén (6 de octubre de 2013). El PAN: liberal y conservador. El Economista. Recuperado de https://www.eleconomista.com.mx/opinion/PAN-liberal-yconservador-20131006-0001.html.

Álvarez, Xochitl (25 de junio de 2018). Alumna de la UG trunca su carrera por abuso e injusticia. El Universal. Recuperado de https://www.eluniversal.com.mx/estados/trunca-su-carrera-por-abuso-e-injusticia

Andréu Abela, Jaime, García Nieto, Antonio y Pérez Corbacho, Ana María. (2007). Evolución de la Teoría Fundamentada como técnica de análisis cualitativo. Madrid: CIS.

Apfelbaum, Erika. (1989). Relaciones de dominación y movimientos de liberación. Un análisis del poder entre los grupos. En José Francisco Morales y Carmen Huici (Eds.), Lecturas de Psicología Social (pp. 261-295). Madrid: UNED. 
Arriola, Carlos. (1994). Ensayos sobre el PAN. Ciudad de México: Porrúa.

Bardales Mendoza, Olga y Ortiz Miguel, Zoila. (2012). Hostigamiento sexual en mujeres y varones universitarios. Estudio exploratorio. Lima: Ministerio de la Mujer y Poblaciones Vulnerables.

Barreto, Magali. (2017). Violencia de género y denuncia pública en la universidad. Revista Mexicana de Sociología, 79(2), 261-286. Recuperado de DOI: http://dx.doi.org/10.22201/iis.01882503p.2017.2.57663

Barreto, Magali. (2018). Testimonio, segunda victimización y reparación. Movilización feminista frente a un caso de violación sexual en la universidad. Sexualidad, salud y sociedad. Revista Latinoamericana, (29), 215-234. Recuperado de https://doi.org/10.1590/1984-6487.sess.2018.29.10.a.

Barreto, Magali y Flores, Natalia. (2016). Institucionalización, violencia de género y demandas desde la base. Nómadas, (44), 201-218. Recuperado de http://www.scielo.org.co/pdf/noma/n44/n44a12.pdf

Bonino, Luis. (2005). Las microviolencias y sus efectos: claves para su detección. En Consuelo Ruiz-Jarabo y Pilar Blanco (Coords.), La violencia contra las mujeres: prevención y detección (pp. 83-102). Madrid: Díaz de Santos.

Buquet Corleto, Ana María. (2016). El orden de género en la educación superior: una aproximación interdisciplinaria. Nómadas, (44), 27-43. Recuperado de https://www.redalyc.org/pdf/1051/105146818003.pdf

Carvajal Olich, Zaira y Delvó Gutiérrez, Patricia. (2009). Costa Rica: un estudio sobre el hostigamiento sexual en población estudiantil universitaria pública y percepción del ambiente homofóbico en 2008. Abra. Revista de la Facultad de Ciencias Sociales 
29(39),

14-30.

Recuperado

de

https://www.revistas.una.ac.cr/index.php/abra/article/view/4122

Casillas, Miguel; Dorantes, Jeysira; Ortiz, Verónica (2017). Estudios sobre la violencia de género en la universidad. Xalapa: Universidad Veracruzana.

Castaño-Castrillón, José Jaime; González, Eliana Katherine; Guzmán, July Andrea; Montoya, Jhon Stiven; Murillo, Juan Manuel; Páez-Cala, Martha Luz; Parra, Luis María; Salazar, Tania Victoria; Velásquez, Yessica. (2010). Acoso sexual en la comunidad estudiantil de la Universidad de Maizales (Colombia). 2008. Estudio de corte transversal. Revista Colombiana de Obstreticia y Ginecología, 61(1), 18-27. Recuperado de http://www.scielo.org.co/pdf/rcog/v61n1/v61n1a03.pdf

Castillo Meraz, Rosalía. (2015). Violencia en las universidades públicas. El caso de la Universidad Autónoma Metropolitana. Ciudad de México: UAM.

Castro, Roberto y Vázquez García, Verónica. (2008). La universidad como espacio de reproducción de la violencia de género. Un estudio de caso en la Universidad Autónoma de Chapingo, México. Estudios Sociológicos, 26(78), 587-616. Recuperado de https://estudiossociologicos.colmex.mx/index.php/es/article/view/314

Centro de Documentación e Información Bolivia, Cedib. (2006). ¿Qué es y cómo se hace análisis de coyuntura? Taller de Análisis de la Realidad. Cochabamba, Bolivia. Recuperado de https://cedib.org/wp-content/uploads/2007/11/modulo12.pdf.

Cerva Cerna, Daniela. (2017). Desafíos para la institucionalización de la perspectiva de género en instituciones de educación superior en México. Una mirada a los contextos organizacionales. Revista Punto Género, (8), 20-38. doi: 10.5354/07190417.2017 .48399 
Cerva, Daniela. (2019). Activismo feminista en las universidades mexicanas: la impronta política de las colectivas de estudiantes ante la violencia contra las mujeres. Ponencia presentada en la Conferencia Policéntrica IFJP-FLACSO, septiembre de 2019.

Evangelista, Angélica. (2017). Hostigamiento y acoso sexual en ámbitos de educación superior del sureste mexicano. Investigação Qualitativa em Ciências Sociais, 3, 336341. Recuperado de https://proceedings.ciaiq.org/index.php/ciaiq2017/article/view/1174

Evangelista, Angélica. (2019). Normalización de la violencia de género como obstáculo metodológico para su comprensión. Nómadas, (51), 85-97. doi: DOI: 10.30578/nomadas.n51a5

Expansión Política (20 de marzo de 2020). Estudiantes de más de 20 universidades de México denuncian a sus acosadores. Expansión. Recuperado de https://politica.expansion.mx/sociedad/2020/03/13/estudiantes-de-universidades-demexico-denuncian-a-acosadores.

Fisher, Bonnie S., Cullen, Francis T. y Turner, Michael G. (2000). The Sexual Victimization of College Women. Rockville: National Institute of Justice.

Fuentes, Carlos. (1959). Las buenas conciencias. Madrid: Club Internacional del Libro.

Gamboa Solís, Flor de María. (2019). Acoso sexual en la Universidad: de protocolos y protocolos. Nómadas, (51), 211-221. doi: 10.30578/nomadas.n51a12

Hensley Choate, Laura. (2011). Sexual Assault Prevention Programs for College Men: An Exploratory Evaluation of the Men Against Violence Model. Journal of College Counseling, (6), 166-176. doi: https://doi.org/10.1002/j.2161-1882.2003.tb00237.x 
Instituto Nacional de Estadística y Geografía, INEGI. (2017). Anuario estadístico y geográfico de Guanajuato 2017. Aguascalientes: Instituto Nacional de Estadística y Geografía. Recuperado de https://www.datatur.sectur.gob.mx/ITxEF_Docs/GTO_ANUARIO_PDF.pdf.

Ley General de Acceso de las Mujeres a una vida libre de Violencia. Diario Oficial de la Federación, 1 de febrero de 2007 (última modificación, 13 de abril de 2018). Recuperado de http://www.diputados.gob.mx/LeyesBiblio/pdf/LGAMVLV_130418.pdf

Mingo, Araceli y Moreno, Hortensia. (2015). El ocioso intento de tapar el sol con un dedo: violencia de género en la universidad. Perfiles educativos, 37(148), 138-155. Recuperado de http://www.scielo.org.mx/pdf/peredu/v37n148/v37n148a9.pdf

Nash, Claudio. (2015). Respuesta institucional ante el acoso sexual en la Universidad de Chile. Santiago de Chile: Universidad de Chile.

Phips. Alison. (2018). Reckoning up: sexual harassment and violence in the neoliberal university. Gender and Education, 32(2), 227-243. doi: https://doi.org/10.1080/09540253.2018.1482413

Programa de las Naciones Unidas para el Desarrollo, PNUD. (2019). Informe de Desarrollo Humano Municipal 2010-2015. Transformando México desde lo local. Ciudad de México: Programa de las Naciones Unidas para el Desarrollo. Recuperado de https://www.mx.undp.org/content/mexico/es/home/library/poverty/informe-dedesarrollo-humano-municipal-2010-2015--transformando-.html

Rionda Ramírez, Jorge Isauro. (2013). Transición de poder en Guanajuato (1991-2013). Tecsistecatl, 5(15), Recuperado de http://www.eumed.net/rev/tecsistecatl/n15/poderguanajuato.html?fbclid=IwAR2jJ6Agg4su2AJBZf4U5LoTOZpLnaZUsyYotmsdf0v4Rosupwx-f8ykyQ 
Rodríguez Gustá, Ana Laura. (2008). Las políticas sensibles al género: variedades conceptuales y desafíos de intervención. Revista Temas y Debates, (16), 109-129. doi:10.35305/tyd.v0i16.75

Trinidad, Antonio, Carrero, Virginia y Soriano, Rosa María. (2006). Teoría fundamentada "Grounded Theory”. La construcción de la teoría a través del análisis interpretacional. Madrid: CIS.

Universidad de Guanajuato (s.f.). Guía Básica si experimentas violencia de género. Recuperado de http:/www.ugto.mx/images/pdf/guia-basica-si-experimentasviolencia-genero-ug-ugto.pdf

Universidad de Guanajuato. (2017). Protocolo de Atención a Casos de Violencia de Género de la Universidad de Guanajuato. Recuperado de http://www.ugto.mx/ugenero/images/pdf/protocolo-de-atencion-a-casos-deviolencia-de-genero-de-la-universidad-de-guanajuato-11-17.pdf

Valls, Rosa, Oliver, Esther; Sánchez-Aroca, Montse; Ruiz, Laura; Melgar, Patricia. (2007). ¿Violencia de género también en las universidades? Investigaciones al respecto. Revista de Investigación Educativa, 25(1), 219-231. Recuperado de https://revistas.um.es/rie/article/view/96771

Varela, Helena. (2020). Las universidades frente a la violencia de género. El alcance limitado de los mecanismos formales. Revista Mexicana de Ciencias Políticas y Sociales, 65(238), 49-80. doi: http://dx.doi.org/10.22201/fcpys.2448492xe.2020.238.68301

Zona Franca (14 de agosto de 2018). Alumnas de la Universidad de Guanajuato denuncian acoso sexual por parte de maestros. Rueda de prensa ofrecida por la Colectiva Alumnas UG en Sororidad, el 14 de agosto de 2018. Recuperado de https://www.youtube.com/watch?v=M-A3mO89LfE 


\section{Sobre la autora}

Helena Varela es doctora en Ciencia Política, Sociología y Antropología Social por la Universidad Autónoma de Madrid, es académica e investigadora de la Universidad Iberoamericana Ciudad de México. Sus líneas de investigación van encaminadas a: los procesos de cambio político, funcionamiento de la democracia, y el papel de las mujeres en procesos políticos y sociales. Entre sus publicaciones recientes destacan: Las universidades frente a la violencia de género. El alcance limitado de los mecanismos formales (2020). Revista Mexicana de Ciencias Políticas y Sociales. Co-coordinadora de: Los indignados mexicanos. Insurgencia juvenil frente al regreso del PRI a la presidencia (2017). Ignacio Ellacuría en las fronteras (2019). 